\title{
Role of electron physics in the development of turbulent magnetic reconnection in collisionless plasmas
}

\author{
W. Daughton ${ }^{1 \star}$, V. Roytershteyn ${ }^{1}$, H. Karimabadi² ${ }^{2}$ L. Yin ${ }^{1}$, B. J. Albright ${ }^{1}$, B. Bergen' and K. J. Bowers ${ }^{1}$
}

\begin{abstract}
Magnetic reconnection releases energy explosively as field lines break and reconnect in plasmas ranging from the Earth's magnetosphere to solar eruptions and astrophysical applications. Collisionless kinetic simulations have shown that this process involves both ion and electron kinetic-scale features, with electron current layers forming nonlinearly during the onset phase and playing an important role in enabling field lines to break $^{\mathbf{1 - 4}}$. In larger two-dimensional studies, these electron current layers become highly extended, which can trigger the formation of secondary magnetic islands ${ }^{5-10}$, but the influence of realistic three-dimensional dynamics remains poorly understood. Here we show that, for the most common type of reconnection layer with a finite guide field, the three-dimensional evolution is dominated by the formation and interaction of helical magnetic structures known as flux ropes. In contrast to previous theories ${ }^{11}$, the majority of flux ropes are produced by secondary instabilities within the electron layers. New flux ropes spontaneously appear within these layers, leading to a turbulent evolution where electron physics plays a central role.
\end{abstract}

Thin current layers are the preferred locations for magnetic reconnection to develop. The most common configuration in nature is guide-field geometry, where the rotation of magnetic field across the layer is less than $180^{\circ}$. Present theoretical ideas of how reconnection proceeds in these configurations are deeply rooted in early analytical work ${ }^{11}$ that, if correct, would imply a direct transition to three-dimensional (3D) turbulence due to a broad spectrum of interacting tearing instabilities. At the core of this idea is the notion that a spectrum of tearing instabilities develops across the initial current sheet for perturbations satisfying the local resonance condition. As these modes grow, the resulting magnetic islands would overlap, leading to stochastic magnetic-field lines and a turbulent evolution. Recently, this type of scenario was proposed as a mechanism for accelerating energetic particles during reconnection ${ }^{12}$. Similar ideas for generating turbulence have been studied in fusion plasmas ${ }^{13}$ using resistive magnetohydrodynamics (MHD) and two-fluid ${ }^{14}$ models. Alternatively, other researchers have imposed turbulent fluctuations within MHD models in an attempt to understand the consequences ${ }^{15}$. In either case, these results are not applicable to the highly collisionless environment of the magnetosphere, where reconnection is initiated within kinetic ion-scale current layers. The ability to study the self-consistent generation of turbulence during magnetic reconnection with first-principles $3 \mathrm{D}$ simulations has only become feasible in the past year.

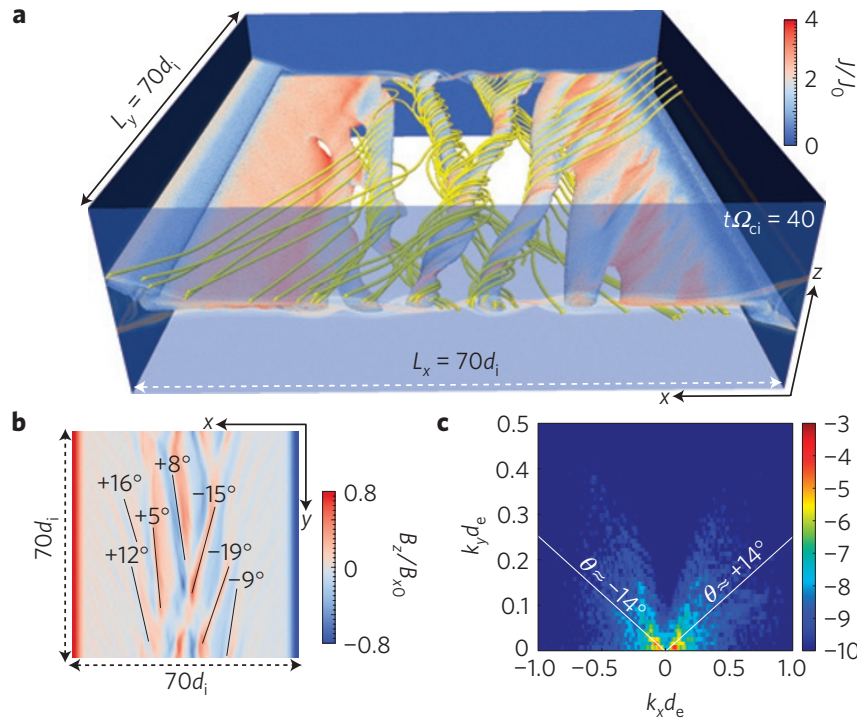

Figure 1 | Formation of primary flux ropes. a, At early time $t \Omega_{\mathrm{ci}}=40$, the tearing instability gives rise to flux ropes as illustrated by an isosurface of the particle density coloured by the magnitude of the current density (normalized by $J_{0} \equiv c B_{x 0} /(4 \pi \lambda)$ ) along with sample magnetic-field lines (yellow). b, Typical angles $\theta \equiv \tan ^{-1}\left(k_{y} / k_{x}\right)$ for these ropes are directly measured by examining $B_{z}$ at the centre of the layer $(z=0)$. $\mathbf{c}$, The power spectrum of $\left|\hat{B}_{z}\right|^{2} / B_{x 0}^{2}$ is shown on a log scale. The solid white line corresponds to the dominant angle in the spectrum.

The 3D simulations were carried out on two petascale supercomputers, Roadrunner and Kraken, using the kinetic particle-in-cell code VPIC (refs 16,17), which solves the relativistic Vlasov-Maxwell system of equations. These capabilities have permitted a series of simulations using over $10^{12}$ computational particles, nearly $10^{3}$ larger than previous two-dimensional (2D) studies ${ }^{5}$. The case described here is initialized with a Harris ${ }^{18}$ current sheet, with magnetic field $\mathbf{B}=B_{x 0} \tanh (z / \lambda) \mathbf{e}_{x}+B_{y 0} \mathbf{e}_{y}$ where $\mathbf{e}_{x}$ and $\mathbf{e}_{y}$ are unit vectors, $\lambda=d_{\mathrm{i}}$ is the initial half-thickness, $d_{\mathrm{i}}$ is an ion inertial length, $B_{y 0}=B_{x 0}$ is a uniform guide field, the ion to electron mass ratio is $m_{\mathrm{i}} / m_{\mathrm{e}}=100$ and further details regarding the set-up are given in the Methods section.

The simulation results in Fig. 1 reveal some dramatic differences from the basic ideas discussed above. First, the concept of magnetic islands in real 3D systems corresponds to extended flux ropes,

\footnotetext{
${ }^{1}$ Los Alamos National Laboratory, Los Alamos, New Mexico 87545, USA, ${ }^{2}$ University of California San Diego, La Jolla, California 92093, USA. *e-mail: daughton@lanl.gov.
} 
which can interact in a variety of complex ways not possible in 2D models. Examples of such complexity were reported in recent experiments involving small flux ropes produced with plasma guns ${ }^{19,20}$. In contrast, the flux ropes shown in Fig. 1 are generated by the tearing instability over a range of oblique angles $\theta<19^{\circ}$, which is substantially less than the expected $\theta<40^{\circ}$ for these parameters ${ }^{11,21}$. Indeed, these results demonstrate a few dominant modes that are localized near the centre of the initial layer. The orientation of these ropes can be directly measured by examining $B_{z}$ near the symmetry plane $(z=0)$ as shown in Fig. $1 \mathrm{~b}$, or by computing the power spectrum of $B_{z}$ over the simulation domain as shown in Fig. 1c. The dominant modes correspond to $k_{x} d_{\mathrm{e}} \approx 0.08$ with $\theta \equiv \tan ^{-1}\left(k_{y} / k_{x}\right) \approx \pm 14^{\circ}$, where $d_{\mathrm{e}}$ is the electron skin depth. Some of the interesting 3D features in Fig. 1 arise from the interaction of these flux ropes, leading to a complex connectivity of magnetic-field lines. However, the observed dynamics at this time is much simpler than expected from previous theories.

Here we reconsider the linear kinetic theory and demonstrate not only that the traditional asymptotic approaches fail dramatically for the oblique tearing modes, but also that realistic predictions can be obtained with numerical techniques. We consider a general electromagnetic perturbation with wavevector $\mathbf{k}=k_{x} \mathbf{e}_{x}+k_{y} \mathbf{e}_{y}$ that satisfies the resonance condition $\mathbf{k} \cdot \mathbf{B}=0$ at a location $z_{s}=-\lambda \tanh ^{-1}\left[k_{y} B_{y 0} /\left(k_{x} B_{x 0}\right)\right]$. The standard approach involves an asymptotic matching between an outer MHD region with a kinetic description within the resonance layer ${ }^{22,23}$. The solution for the outer region features a discontinuity in the first derivative of the perturbed magnetic field $\tilde{B}_{z}$ across the resonance layer, which captures the destabilizing influence of magnetic shear that drives tearing. However, previous theories ${ }^{11}$ used an incorrect equation for the outer region, which does not recover MHD (ref. 24) for the oblique modes. Correcting for this error, we obtain

$$
\begin{aligned}
\Delta^{\prime} & =\lim _{\epsilon \rightarrow 0}\left[\left(\frac{\tilde{B}_{z}^{\prime}}{\tilde{B}_{z}}\right)_{z_{s}+\epsilon}-\left(\frac{\tilde{B}_{z}^{\prime}}{\tilde{B}_{z}}\right)_{z_{s}-\epsilon}\right] \\
& \approx \frac{2}{\lambda}\left(\frac{1}{k \lambda}-k \lambda\right)\left[1+\frac{\tanh ^{2}\left(z_{s} / \lambda\right)}{2}\left(1+\frac{1}{1-k \lambda}\right)\right]
\end{aligned}
$$

where $k \equiv|\mathbf{k}|$ and $\tilde{B}_{z}$ is the perturbed magnetic field. The term in brackets causes $\Delta^{\prime}$ to increase for modes localized on the edge of the layer $\tanh \left[z_{s} / \lambda\right]=\tan (\theta) B_{y 0} / B_{x 0}$, corresponding to oblique angle $\theta \equiv \tan ^{-1}\left(k_{y} / k_{x}\right)$. Using this solution for $\Delta^{\prime}$ and neglecting the electrostatic perturbation within the resonance layer results in growth rate and real frequency

$$
\begin{gathered}
\frac{\gamma}{\Omega_{\mathrm{ci}}} \approx \frac{1}{\sqrt{\pi}} \frac{\alpha}{1+\alpha}\left(\frac{\rho_{\mathrm{i}}}{\lambda}\right)^{3}\left(1+\frac{T_{\mathrm{e}}}{T_{\mathrm{i}}}\right)\left[1-(k \lambda)^{2}\right] \frac{B_{x 0}}{B_{y 0}} \mathcal{G} \\
\omega_{\mathrm{r}} \approx k_{y} U_{\mathrm{e}} \frac{\left[1-\alpha T_{\mathrm{e}} / T_{\mathrm{i}}\right]}{1+\alpha} \frac{1}{1+\hat{n}_{\mathrm{b}} \cosh ^{2}\left(z_{s} / \lambda\right)}
\end{gathered}
$$

where $\rho_{\mathrm{i}}=v_{t h_{\mathrm{i}}} / \Omega_{\mathrm{ci}}, \quad v_{t h_{\mathrm{i}}}=\left(2 T_{\mathrm{i}} / m_{\mathrm{i}}\right)^{1 / 2}, \quad \Omega_{\mathrm{ci}} \equiv e B_{x 0} /\left(m_{\mathrm{i}} c\right), \quad U_{\mathrm{e}}=$ $2 c T_{\mathrm{e}} /\left(e B_{x 0} \lambda\right)$ is the electron drift velocity, $\alpha \equiv\left[T_{\mathrm{e}} m_{\mathrm{e}} /\left(T_{\mathrm{i}} m_{\mathrm{i}}\right)\right]^{1 / 2}$ and the angular dependence of the growth rate is

$$
\mathcal{G}=\left[1-\sin ^{2}(\theta)\left(1-\left(\frac{B_{y 0}}{B_{x 0}}\right)^{2} \frac{1-k \lambda / 2}{1-k \lambda}\right)\right] \frac{1}{1+\hat{n}_{\mathrm{b}} \cosh ^{2}\left(z_{s} / \lambda\right)}
$$

For weak background density $\hat{n}_{\mathrm{b}} \equiv n_{\mathrm{b}} / n_{0} \ll 1$, equation ( 1 ) implies that the fastest-growing modes are oblique for $B_{y 0} / B_{x 0}>((1-$ $k \lambda) /(1-k \lambda / 2))^{1 / 2}$. Even before this condition is reached, modes are destabilized for $\Delta^{\prime}>0$, corresponding to angles up to $\theta_{\max }=\tan ^{-1}\left(B_{x 0} / B_{y 0}\right)$.

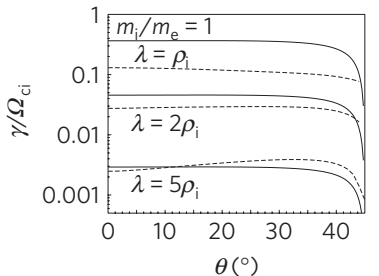

C

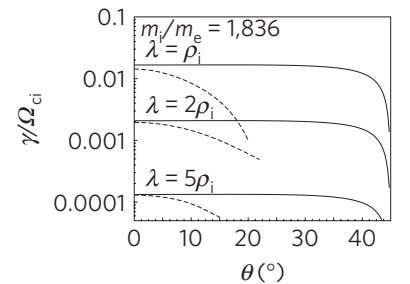

b

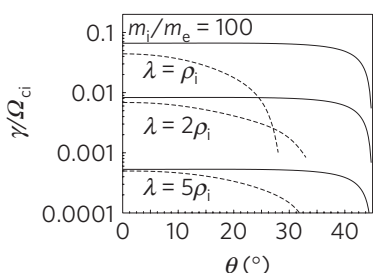

d

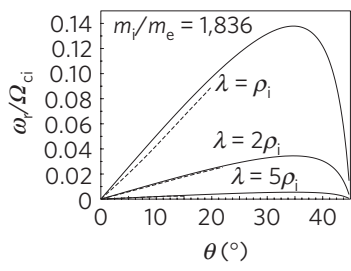

Figure 2 | Theoretical predictions for oblique tearing instability. The asymptotic theory (solid) from equation (1) is compared with the exact Vlasov results ${ }^{25}$ (dashed) as a function of oblique angle $\theta=\tan ^{-1}\left(k_{y} / k_{x}\right)$ for the mass ratio $m_{\mathrm{i}} / m_{\mathrm{e}}$ and sheet thickness $\lambda$ indicated. $\mathbf{a}-\mathbf{c}$, The growth rate; $\mathbf{d}$, the real frequency corresponding to $\mathbf{c}$. Other parameters are held fixed, $k \lambda=0.4, B_{y 0}=B_{x 0}, T_{\mathrm{i}}=T_{\mathrm{e}}$ and $n_{\mathrm{b}}=0.3 n_{0}$.

Even with the corrected $\Delta^{\prime}$, these results are in clear disagreement with Fig. 1, because the predicted range of angles $\theta_{\max }= \pm 45^{\circ}$ is much broader than actually observed. However, the asymptotic theory is based on the ordering $\lambda \gg \rho_{\mathrm{i}}$, whereas our focus is ion-scale layers $\lambda \sim \rho_{\mathrm{i}}$, where the onset of reconnection is thought to occur in magnetospheric plasmas. To treat this regime rigorously, we employ an exact numerical approach which solves the linearized Vlasov-Maxwell system ${ }^{25,26}$. As shown in Fig. 2, these exact growth rates are in good agreement with equation (1) for the limit of an electron-positron plasma $m_{\mathrm{i}}=m_{\mathrm{e}}$ at all angles. At high mass ratio, equation (1) also works reasonably well for modes with $k_{y}=0$, and the agreement improves for thicker current sheets where the asymptotic theory is better justified. However, the asymptotic theory fails dramatically at oblique angles, even for the thicker layers. The exact Vlasov results predict that the unstable modes are limited to $\theta_{\max }<20^{\circ}$ for $m_{\mathrm{i}} / m_{\mathrm{e}}=100$, in good agreement with the simulation results in Fig. 1. Since the range of unstable modes $\theta_{\max }<15^{\circ}$ in a hydrogen plasma is quite similar, these results provide a nice justification for the reduced mass ratio employed in this letter. The real frequency in Fig. $2 \mathrm{~d}$ is also a bit lower than expected from equation (1), but the dramatic failure of the asymptotic theory is not well understood. We note that the eigenfunction obtained for oblique modes with $m_{\mathrm{i}} / m_{\mathrm{e}} \gg 1$ is complex, with a rapid change in phase across the ion resonance layer, a feature that is inconsistent with the asymptotic matching.

For parameter regimes relevant to magnetospheric plasmas, these results demonstrate that the spectrum of oblique tearing modes is much narrower than previous expectations, which implies that the generation of turbulence by overlapping magnetic islands cannot proceed as previously thought. Although the narrow spectrum of modes persists even for thicker current sheets $\lambda=5 \rho_{\mathrm{i}}$, it remains unclear if this holds for $\lambda \gg \rho_{\mathrm{i}}$ or stronger guide fields $B_{y 0} \gg B_{x 0}$. However, over longer timescales these simulations reveal a new scenario in which flux ropes can be generated through secondary instabilities in the highly extended electron-scale current sheets that form nonlinearly during the onset of reconnection. These electron layers are a general feature of fast reconnection in collisionless plasmas and play a crucial role in breaking the frozen-in condition $^{1-4}$. In sufficiently large systems, previous $2 \mathrm{D}$ simulations have demonstrated that these layers may become highly extended and form secondary magnetic islands ${ }^{5-10}$. However, the manner in which this can occur is vastly expanded in three dimensions. 
a

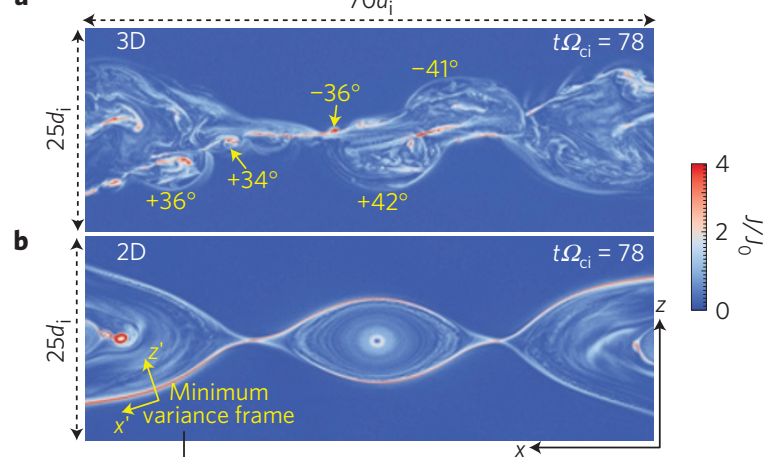

c

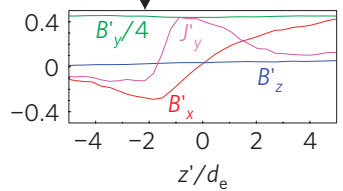

d

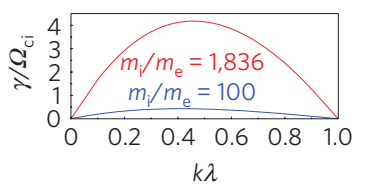

Figure 3 | Formation of secondary flux ropes. $\mathbf{a}, \mathbf{b}$, Slice of the current density at $y=35 d_{i}$ from the $3 D$ simulation (a) compared with the corresponding $2 \mathrm{D}$ result $(\mathbf{b})$. c, The structure of the separatix layer at the location indicated. Profiles in $\mathbf{c}$ are shown in the minimum-variance frame ( $18^{\circ}$ rotation about $y$ followed by $52^{\circ}$ rotation about $\left.z^{\prime}\right)$. d, Fitting to a Harris profile gives a half-thickness $\lambda \approx 2 d_{\mathrm{e}}$ with guide field $B_{y}^{\prime} \approx 4.4 B_{x 0}^{\prime}$ resulting in the growth rate shown. e, The power spectrum $\left|\hat{B}_{z}\right|^{2} / B_{x 0}^{2}$ for the $3 D$ simulation on a log scale. The solid white line corresponds to the dominant angle, whereas the dashed line is the simple estimate from c.

To illustrate this point, Fig. 3 compares a slice of the current density between the $3 \mathrm{D}$ and a corresponding $2 \mathrm{D}$ case at time $t \Omega_{\mathrm{ci}}=78$. Whereas the current layers along the separatrices are stable in two dimensions, these layers are violently unstable in three dimensions to the formation of flux ropes over a wide range of oblique angles as shown in Fig. 3a, causing the current density to become highly filamented and time dependent.

It seems that these dramatic differences between two and three dimensions are due to tearing-type instabilities driven by the strong magnetic shear across the electron-scale layers. To demonstrate this mechanism, the profiles of the current density and magnetic field are shown in Fig. $3 \mathrm{c}$ across the separatrix layer in the $2 \mathrm{D}$ simulation. Fitting the profiles to a local Harris sheet corresponds to $\lambda \approx 2 d_{\mathrm{e}}$, with a magnetic shear angle of $26^{\circ}$ across the layer. The kinetic theory $^{26}$ for these parameters in Fig. $3 \mathrm{~d}$ predicts $\gamma / \Omega_{\mathrm{ci}} \approx 0.42$ for the fastest-growing modes with $k d_{\mathrm{e}} \approx 0.2$. At realistic hydrogen mass ratio, the growth rate increases to $\gamma / \Omega_{\mathrm{ci}} \approx 4.2$ assuming that the layer thickness remains on the scale $\sim 2 d_{\mathrm{e}}$. The separatrix current layer in Fig. $3 \mathrm{~b}$ from the left boundary to the first X line is approximately $\sim 220 d_{\mathrm{e}}$ long, which implies it should break up into seven filaments, whereas there are four rope structures visible in Fig. 3a. However, this separatrix layer actually begins to break up at time $t \Omega_{\mathrm{ci}} \approx 60$, and originally forms six filaments. Thus some of these structures have already coalesced by time $t \Omega_{\mathrm{ci}} \approx 78$ shown in Fig. 3. The angle of the modes is determined by $\mathbf{k} \cdot \mathbf{B}=0$ near the centre of the electron layer at the time of break-up. The sample profiles from the $2 \mathrm{D}$ case in Fig. $3 \mathrm{~b}, \mathrm{c}$ correspond to $\theta \approx 52^{\circ}$, which is larger than the observed angles for these structures in Fig. 3a. Again, exact agreement is not expected because filamentary structures begin forming at earlier time in the $3 \mathrm{D}$ simulation. To better quantify the structure of the magnetic-field perturbations,
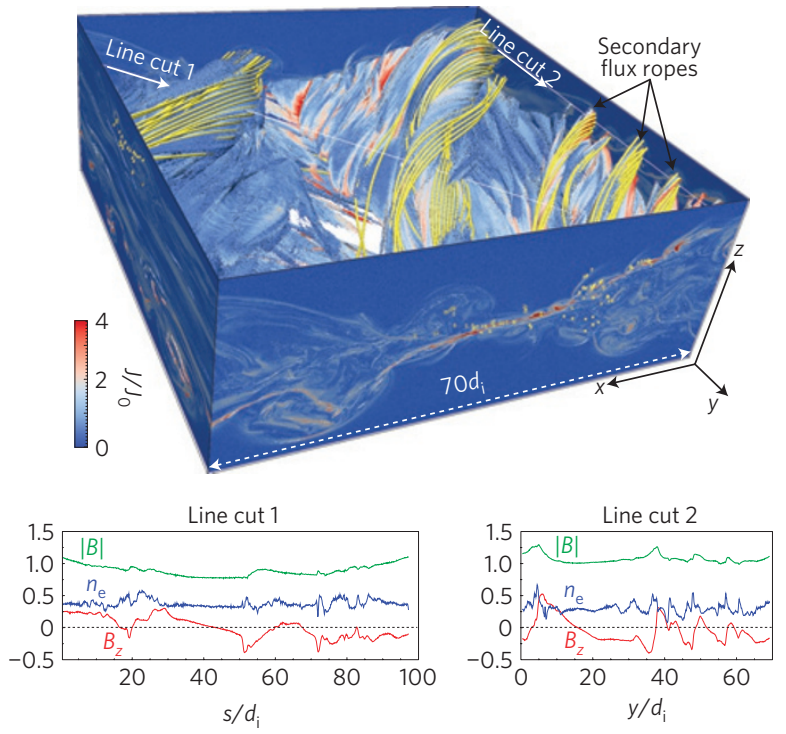

Figure 4 | Development of turbulent reconnection. At late time $t \Omega_{\mathrm{ci}}=98$, the secondary flux ropes have grown to large amplitudes and interact over a wide range of angles, giving rise to a turbulent evolution. The 3D structure is illustrated by an isosurface of particle density coloured by current density, along with selected magnetic-field lines (yellow). To illustrate the observational signatures of these flux ropes, the normal magnetic field $B_{z}$, total magnetic field $|\mathbf{B}|$ and electron density $n_{\mathrm{e}}$ are plotted along two trajectories.

the power spectrum of $B_{z}$ is given in Fig. 3e. The form of the spectrum is much broader in $k$ space and indicates the presence of highly anisotropic narrow structures in $B_{z}$, which are signatures of the flux ropes. The peak power occurs for $\theta \approx \pm 34^{\circ}$, but there is actually significant power out to $\theta \approx 52^{\circ}$ consistent with the above estimate. On the basis of these results, it seems that the growth time, wavelength and angle of these structures are consistent with tearing instabilities in the electron layers.

The $3 \mathrm{D}$ structure of the turbulent reconnection is illustrated in Fig. 4 at somewhat later time $t \Omega_{\mathrm{ci}}=98$, where the oblique flux ropes have grown to larger amplitude and the helical magneticfield structure of the ropes is clearly visible. The simulation is dominated by the interaction of highly anisotropic structures across multiple scales, including electron-scale current sheets that continually reform and break up into filaments, along with flux ropes generated at these scales and quickly growing well above ion scales. This turbulence is highly inhomogeneous and is continually self-generated within the reconnection layer, which in the present study is embedded in an otherwise laminar plasma.

These results have immediate implications for spacecraft observations of magnetic reconnection. In the solar wind, observational studies of reconnection ${ }^{27}$ are based on an assumed, idealized, 2D geometry with flux ropes centred in the reconnection exhaust. Neither of these assumptions is consistent with the structure observed in Fig. 4. Our results indicate the need to expand the identification criteria to cover the more complex behaviour reported here. Indeed, whereas many examples of essentially 2D laminar exhausts have been reported ${ }^{27}$, other cases indicate that more complicated features are present ${ }^{28}$. However, essentially all observations of reconnection in the solar wind are $>1,000 d_{\mathrm{i}}$ downstream from an active $\mathrm{X}$ line, and it remains unclear from our initial study how far downstream this turbulence might persist in these extremely large systems.

At the magnetopause, flux ropes are commonly referred to as flux transfer events ${ }^{29}$ and may play an important role in transport across the magnetopause. However, their formation mechanism and precise signatures remain under debate. Of the leading ideas ${ }^{29-31}$, 
these simulations are more consistent with the multiple-X-line model $^{30}$ rather than the single-X-line model ${ }^{31}$, but with important differences. Observationally, the degree of asymmetry in the bipolar magnetic field $B_{z}$ is an important signature for identifying flux transfer events and distinguishing between models. For example, the nearly symmetric bipolar $B_{z}$ signature in the bottom right panel of Fig. 4 is generally consistent with the multiple-X-line mode ${ }^{32,33}$. However, a different trajectory through these same structures (left panel) shows strong asymmetries in the bipolar $B_{z}$, which researchers might interpret in terms of the single-Xline model ${ }^{32,33}$. Clearly, the actual $3 \mathrm{D}$ structure in Fig. 4 is more complicated than the simple models currently employed, which implies that many flux ropes may not have been properly recognized in previous studies. These results are important for the upcoming Magnetospheric Multiscale Mission, which is focused on the role of electron physics in collisionless reconnection. The high-timeresolution diagnostics on this mission will enable measurements of the extended electron layers as they break up into flux ropes near reconnection sites.

\section{Methods}

The simulation is initialized with a Harris ${ }^{18}$ current sheet and, to better mimic a large open system, open boundary conditions ${ }^{5}$ are employed in the $x$ and $z$ directions with periodic boundary conditions in the $y$ direction. Following previous $2 \mathrm{D}$ studies ${ }^{5}$, a weak $4 \%$ magnetic perturbation is included to set up a large-scale flow pattern consistent with the open boundaries, while still enabling linear modes to grow. The initial density profile is $n(z)=n_{0} \operatorname{sech}^{2}(z / \lambda)+n_{\mathrm{b}}$, where $n_{0}$ is the central Harris density and $n_{\mathrm{b}}$ is a uniform background density. Lengths are normalized by the ion $d_{\mathrm{i}}=c / \omega_{\mathrm{pi}}$ and electron $d_{\mathrm{e}}=c / \omega_{\mathrm{pe}}$ inertial scales, where $\omega_{\mathrm{ps}}=\left(4 \pi e^{2} n_{0} / m_{s}\right)^{1 / 2}$ for each species $s=\mathrm{i}$, e. The domain size is $L_{x} \times L_{y} \times L_{z}=70 d_{\mathrm{i}} \times 70 d_{\mathrm{i}} \times 35 d_{\mathrm{i}}$ with $2,048 \times 2,048 \times 1,024$ cells. The simulation used 120 particles per cell for each species, corresponding to a total of $\sim 10^{12}$ particles. Other parameters are $\lambda=d_{\mathrm{i}}, m_{\mathrm{i}} / m_{\mathrm{e}}=100, B_{y 0}=B_{x 0}$, $T_{\mathrm{i}}=T_{\mathrm{e}}$ and $\omega_{\mathrm{pe}} / \Omega_{\mathrm{ce}}=2$ where $\Omega_{\mathrm{ce}}=e B_{x 0} /\left(m_{\mathrm{e}} c\right)$. To ensure that magnetic flux enters the system in a controlled fashion, the open boundary conditions were modified on the $z$ boundaries to uniformly drive a prescribed inflow $U_{z}=0.08 V_{\mathrm{A}}[1-\exp (-t / \tau)]$ where $V_{\mathrm{A}}=B_{x 0} / \sqrt{4 \pi m_{\mathrm{i}} n_{\mathrm{b}}}$ is the Alfvén velocity and $\tau \Omega_{\mathrm{ci}}=80$. The spectra in Figs 1 and 3 were computed with a discrete Fourier transform of $B_{z}$ in all three spatial directions. In both the $z$ and $x$ directions a Blackman window ${ }^{34}$ was employed to suppress spurious features in the spectrum from the non-periodic boundary conditions. Finally, the reduced spectrum $\hat{B}_{z}\left(k_{x}, k_{y}\right)$ corresponds to a sum over $k_{z}$.

\section{Received 25 October 2010; accepted 4 March 2011; published online 10 April 2011}

\section{References}

1. Hesse, M., Schindler, K., Birn, J. \& Kuznetsova, M. The diffusion region in collisionless magnetic reconnection. Phys. Plasmas 6, 1781-1795 (1999).

2. Pritchett, P. Geospace environmental modeling magnetic reconnection challenge: Simulations with a full particle electromagnetic code. J. Geophys. Res. 106, 3783-3798 (2001).

3. Pritchett, P. Onset and saturation of guide-field magnetic reconnection. Phys. Plasmas 12, 062301 (2005).

4. Ricci, P., Brackbill, J., Daughton, W. \& Lapenta, G. Collisionless magnetic reconnection in the presence of a guide field. Phys. Plasmas 11, 4102-4114 (2004).

5. Daughton, W., Scudder, J. \& Karimabadi, H. Fully kinetic simulations of undriven magnetic reconnection with open boundary conditions. Phys. Plasmas 13, 072101 (2006).

6. Drake, J., Swisdak, M., Schoeffler, K., Rogers, B. \& Kobayashi, S. Formation of secondary islands during magnetic reconnection. Geophys. Res. Lett. 33, L13105 (2006).

7. Klimas, A., Hesse, M. \& Zenitani, S. Particle-in-cell simulations of collisionless reconnection with open outflow boundaries. Phys. Plasmas 15, 082102 (2008).

8. Karimabadi, H., Daughton, W. \& Scudder, J. Multi-scale structure of the electron diffusion region. Geophys. Res. Lett. 34, L13104 (2007).

9. Karimabadi, H., Roytershteyn, V., Mouikis, C., Kistler, L. \& Daughton, W. Flushing effect in reconnection: Effects of minority species of oxygen ions. Planet. Space Sci. doi:10.1016/j.pss.2010.07.014 (2010).

10. Daughton, W. et al. in AIP Conference Proceedings: Modern Challenges in Nonlinear Plasma Physics Vol. 1320 (eds Vassiliadis, D., Fung, S., Shao, X., Daglis, I. \& Huba, J.) 144-159 (2011).

11. Galeev, A., Kuznetsova, M. \& Zelenyi, L. Magnetopause stability threshold for patchy reconnection. Space Sci. Rev. 44, 1-41 (1986).
12. Drake, J. F., Swisdak, M., Che, H. \& Shay, M. A. Electron acceleration from contracting magnetic islands during reconnection. Nature 443, 553-556 (2006).

13. Carreras, B., Hicks, H., Holmes, J. \& Waddell, B. Nonlinear coupling of tearing modes with self-consistent resistivity evolution in tokamaks. Phys. Fluids 23, $1811-1826$ (1980).

14. Borgogno, D., Grasso, D., Califano, F. P. F., Pegoraro, F. \& Farina, D. Aspects of three-dimensional magnetic reconnection. Phys. Plasmas 12, 032309 (2005)

15. Kowal, G., Lazarian, A., Vishniac, E. T. \& Otmianowska-Mazur, K. Numerical tests of fast reconnection in weakly stochastic magnetic fields. Astrophys. J. 700, $63-85$ (2009).

16. Bowers, K. J., Albright, B. J., Yin, L., Bergen, B. \& Kwan, T. J. T. Ultrahigh performance three-dimensional electromagnetic relativistic kinetic plasma simulation. Phys. Plasmas 15, 055703 (2008).

17. Bowers, K. et al. Advances in petascale kinetic simulations with VPIC and Roadrunner. J. Phys.: Conf. Series 180, 012055 (2009).

18. Harris, E. G. On a plasma sheath separating regions of oppositely directed magnetic field. Nuovo Cimento. 23, 115-121 (1962).

19. Intrator, T., Sun, X., Lapenta, G., Dorf, L. \& Furno, I. Experimental onset threshold and magnetic pressure pile-up for 3D reconnection. Nature Phys. 5, 521-526 (2009).

20. Lawrence, E. \& Gekelman, W. Identification of a quasiseparatrix layer in a reconnecting laboratory magnetoplasma. Phys. Rev. Lett. 103, 105002 (2009).

21. Quest, K. B. \& Coroniti, F. Tearing at the dayside magnetopause. J. Geophys. Res. 86, 3289-3298 (1981).

22. Drake, J. \& Lee, Y. Kinetic theory of tearing instabilities. Phys. Fluids 20, 1341-1353 (1977).

23. Coppi, B., Mark, J.W-K., Sugiyma, L. \& Bertin, G. Reconnecting modes in collisionless plasmas. Phys. Rev. Lett. 42, 1058-1061 (1979).

24. Furth, H., Killeen, J. \& Rosenbluth, M. Finite-resistivity instabilities of a sheet pinch. Phys. Fluids 1963, 459-484 (1963).

25. Daughton, W. Electromagnetic properties of the lower-hybrid drift instability in a thin current sheet. Phys. Plasmas 10, 3103-3119 (2003).

26. Daughton, W. \& Karimabadi, H. Kinetic theory of collisionless tearing at the magnetopause. J. Geophys. Res. 110, A03217 (2005).

27. Gosling, J. Twelfth International Solar Wind Conference Vol. 1216, 188-193 (2010).

28. Gosling, J. et al. Direct evidence for prolonged magnetic reconnection at a continuous x-line within the heliospheric current sheet. Geophys. Res. Lett. 34, L06102 (2007).

29. Russell, C. \& Elphic, R. C. Initial ISEE magnetometer results: Magnetopause observations. Space Sci. Rev. 22, 681-715 (1978).

30. Lee, L. \& Fu, Z. F. A theory of magnetic flux transfer at the Earths magnetopause. Geophys. Res. Lett. 12, 105-108 (1985).

31. Scholer, M. Magnetic flux transfer at the magnetopause based on single X-line bursty reconnection. Geophys. Res. Lett. 15, 291-294 (1988).

32. Ku, H. \& Sibeck, D. Flux transfer events produced by the onset of merging at multiple X-lines. J. Geophys. Res. 105, 2657-2675 (2000).

33. Fear, R. et al. The azimuthal extent of three flux transfer events. Ann. Geophys. 26, 2353-2369 (2008).

34. Oppenheim, A. \& Schafer, R. Discrete-Time Signal Processing (Prentice-Hall, 1999).

\section{Acknowledgements}

We gratefully acknowledge support from the US Department of Energy through the LANL/LDRD Program and through the Advanced Simulation and Computing program for access to Roadrunner computing resources. Simulations carried out on Kraken were supported by an allocation of advanced computing resources provided by the National Science Foundation at the National Institute for Computational Sciences (http://www.nics.tennessee.edu/). Contributions from H.K. were supported by NASA through the Heliophysics Theory Program and NSF through ATM 0802380. We thank K. Quest and J. T. Gosling for discussions and P. Fasel, J. Patchett, J. Ahrens, B. Loring, B. Geveci and D. Partyka for assistance with interfacing the simulation data with ParaView visualization software.

\section{Author contributions}

W.D. carried out the kinetic simulations and wrote the paper. W.D., V.R. and H.K. carried out the analytic theory. V.R. carried out the spectral analysis. K.J.B. originally developed the VPIC code and L.Y., B.J.A., B.B. and K.J.B. ported and tested the VPIC code for petascale supercomputers. All of the authors discussed the results and commented on the paper.

\section{Additional information}

The authors declare no competing financial interests. Reprints and permissions information is available online at http://npg.nature.com/reprintsandpermissions. Correspondence and requests for materials should be addressed to W.D. 